\title{
POLYNOMIAL GLOBAL PRODUCT STRUCTURE
}

\author{
ANDY HAMMERLINDL \\ (Communicated by Nimish Shah)
}

\begin{abstract}
An Anosov diffeomorphism is topologically conjugate to an infranilmanifold automorphism if and only if it has polynomial Global Product Structure.
\end{abstract}

\section{INTRODUCTION}

A famous conjecture states that every Anosov diffeomorphism is topologically conjugate to an algebraic example, specifically an infranilmanifold automorphism. In classification results toward this end ([5], 8], [15]), a key step is establishing Global Product Structure.

Foliations $\mathcal{F}$ and $\mathcal{G}$ have Global Product Structure if every leaf $\mathcal{F}(x)$ intersects every leaf $\mathcal{G}(y)$ in a unique point $[x, y]$. An Anosov diffeomorphism has Global Product Structure if its stable and unstable foliations have Global Product Structure on the universal cover. While it is not clear if Global Product Structure alone is enough to give a classification, the following stronger condition is.

Foliations $\mathcal{F}$ and $\mathcal{G}$ have polynomial Global Product Structure if there is a polynomial $p$ such that $d_{\mathcal{F}}(x,[x, y])+d_{\mathcal{G}}(y,[x, y])<p(d(x, y))$ for all $x$ and $y$. Here, $d_{\mathcal{F}}$ and $d_{\mathcal{G}}$ are distances measured along the leaves, and $d$ is distance on the ambient manifold.

Theorem 1.1. An Anosov diffeomorphism is topologically conjugate to an infranilmanifold automorphism if and only if it has polynomial Global Product Structure.

In particular, all known examples of Anosov systems have polynomial Global Product Structure. The major ingredients needed to prove the "if" direction appear in the work of Brin and Manning [3, 5, and the "only if" direction is an exercise in basic Lie group theory. However, the result seems to have gone unnoticed to date.

We give a specific application of the result. A foliation $\mathcal{F}$ is quasi-isometric if, after lifting to the universal cover, there is $Q>1$ such that $d_{\mathcal{F}}(x, y) \leq Q d(x, y)+Q$ for all $x$ and $y$ on the same lifted leaf.

Theorem 1.2. An Anosov diffeomorphism with quasi-isometric stable and unstable foliations is topologically conjugate to an infranilmanifold automorphism.

The same analysis applies to partially hyperbolic systems and we give several results, including the following.

Received by the editors October 1, 2012 and, in revised form, January 29, 2013.

2010 Mathematics Subject Classification. Primary 37D20, 37D30. 
Theorem 1.3. For a (strongly) partially hyperbolic diffeomorphism on a 3-manifold $M$, the stable, center, and unstable foliations exist and are quasi-isometric if and only if $M$ is finitely covered by the 3-torus.

\section{Anosov systems}

Definition. Consider a diffeomorphism $f$ on a compact Riemannian manifold $M$ with a $T f$-invariant splitting $T M=E^{s} \oplus E^{c} \oplus E^{u}$ and constants $\sigma<1<\mu$ such that

$$
\left\|T f v^{s}\right\|<\sigma<\left\|T f v^{c}\right\|<\mu<\left\|T f v^{u}\right\|
$$

for all unit vectors $v^{s} \in E^{s}, v^{c} \in E^{c}$, and $v^{u} \in E^{u}$.

- If $E^{c}$ is the zero bundle, then $f$ is called Anosov.

- If exactly one of $E^{s}$ and $E^{u}$ is the zero bundle, then $f$ is called weakly partially hyperbolic.

- If all three subbundles are non-zero, then $f$ is called strongly partially hyperbolic.

We first give two polynomial estimates which hold for all Anosov systems.

Definition. For an ordered pair of foliations $(\mathcal{F}, \mathcal{G})$, an $R$-rectangle is a continuous map $\phi:[0,1]^{2} \rightarrow M$ such that each $\phi([0,1] \times\{v\})$ lies in a leaf of $\mathcal{F}$, each $\phi(\{t\} \times$ $[0,1])$ lies in a leaf of $\mathcal{G}$, and $\sup \left\{d_{\mathcal{G}}\left(\phi\left(t, v_{1}\right), \phi\left(t, v_{2}\right)\right): t, v_{1}, v_{2} \in[0,1]\right\}<R$.

The pair $(\mathcal{F}, \mathcal{G})$ has polynomial bounds on rectangles if there is a polynomial $p$ such that

$$
\sup _{t} d_{\mathcal{F}}(\phi(0,0), \phi(t, 0))<\frac{1}{p(R)} \Rightarrow d_{\mathcal{F}}(\phi(0,1), \phi(1,1))<1
$$

for every $R$-rectangle $\phi$. We simply write that $\mathcal{F}$ and $\mathcal{G}$ have polynomial bounds on rectangles, if both $(\mathcal{F}, \mathcal{G})$ and $(\mathcal{G}, \mathcal{F})$ satisfy the property.

Notation. In what follows, a lifted foliation means the lift of a foliation from a compact manifold to its universal cover.

The following observation is at the core of the proof in [2].

Lemma 2.1. For an Anosov diffeomorphism, the lifted stable and unstable foliations have polynomial bounds on rectangles.

Appendix $\mathrm{A}$ contains a proof.

Definition. A Riemannian manifold $\tilde{M}$ has polynomial growth of volume, if there is a polynomial $p$ such that volume $(B(x, R))<p(R)$ for all $x \in \tilde{M}$ and $R>0$. Here $B(x, R)$ is the set of all points at a distance less than $R$ from $x$.

A foliation $\mathcal{F}$ has polynomial growth of volume if there is a polynomial $p$ such that volume $(\mathcal{F}(x, R))<p(R)$ for all $x \in \tilde{M}$ and $R>0$, and where $\mathcal{F}(x, R)$ denotes all points reachable from $x$ by a path in $\mathcal{F}(x)$ of length less than $R$. (That is, each leaf has polynomial growth, and the polynomial is independent of the leaf.)

If a map $f: M \rightarrow M$ is uniformly expanding, the universal cover $\tilde{M}$ must have polynomial growth of volume [17. The same applies to the uniform contraction or expansion inside a stable or unstable foliation.

Lemma 2.2. In an Anosov (or partially hyperbolic) diffeomorphism, the lifted stable and unstable foliations have polynomial growth of volume. 
Brin showed that for an Anosov diffeomorphism with "pinched" spectrum, the supporting manifold has a universal cover with polynomial growth of volume [3]. The proof relies on the following.

Proposition 2.3. Suppose $\mathcal{F}$ and $\mathcal{G}$ are $C^{0}$ foliations with $C^{1}$ leaves on a compact Riemannian manifold $M$, such that $T \mathcal{F}$ and $T \mathcal{G}$ are continuous as subbundles of $T M$. If the lifted foliations

- have polynomial Global Product Structure,

- have polynomial bounds on rectangles, and

- have polynomial growth of volume,

then the universal cover has polynomial growth of volume.

Pinching is needed only to establish the first of the above three conditions.

Corollary 2.4. If an Anosov diffeomorphism $f: M \rightarrow M$ has polynomial Global Product Structure, the universal cover has polynomial growth of volume.

For a compact manifold $M$, the universal cover has polynomial growth of volume if and only if the fundamental group does. In such a case, a famous result of Gromov shows that the fundamental group is virtually nilpotent [9]. This fact allowed Brin and Manning to classify Anosov diffeomorphisms with pinched spectra [5]. Their paper never directly uses the hypothesis of pinching and actually proves the following more general result.

Theorem 2.5 (Brin-Manning). If an Anosov diffeomorphism $f$ has Global Product Structure, and the universal cover has polynomial growth of volume, then $f$ is topologically conjugate to an infranilmanifold automorphism.

Taken with Corollary 2.4, this proves one direction of Theorem 1.1. The other direction is an enjoyable exercise in basic Lie group theory. Appendix B gives hints. The proof of Theorem 2.5 given in [5] relies on a result stated by Auslander [1] now known to be false (7], 14]). Appendix C] shows how to handle this issue.

Definition. We define linear Global Product Structure as polynomial Global Product Structure where the associated polynomial may be taken as linear, that is, of the form $p(t)=a t+b$.

The following is proved, but not explicitly stated in [12].

Proposition 2.6. An Anosov diffeomorphism with quasi-isometric stable and unstable foliations has linear Global Product Structure.

Taken with Theorem 1.1 this proves Theorem 1.2

\section{Partially hyperbolic Systems}

First, consider weakly partially hyperbolic systems. For simplicity, assume $E^{s}$ is the zero bundle. That is, the splitting is $T M=E^{c} \oplus E^{u}$. For every weakly partially hyperbolic system, there is a unique foliation tangent to the unstable subbundle $E^{u}$. For the center subbundle $E^{c}$, there may or may not be a tangent foliation. It is not known if such a center foliation must be unique [6].

Definition. A weakly partially hyperbolic system has (linear/polynomial) Global Product Structure if there are foliations $W^{c}$ and $W^{u}$ tangent to $E^{c}$ and $E^{u}$ which satisfy the property after lifting to the universal cover. 
The following is proved in Appendix A.

Lemma 3.1. If a weakly partially hyperbolic diffeomorphism has linear Global Product Structure, then the lifted foliations $W^{c}$ and $W^{u}$ have polynomial bounds on rectangles.

As in the Anosov case, the strong expansion in the unstable direction implies that $W^{u}$ has polynomial growth of volume, but no such claim can be made for the center. Lemma 3.1 and Proposition 2.3 give the following.

Proposition 3.2. If $f: M \rightarrow M$ is weakly partially hyperbolic with linear Global Product Structure, and the lifted foliation $W^{c}$ has polynomial growth of volume, then the universal cover has polynomial growth of volume.

Proposition 2.6 also applies to the foliations of a weakly partially hyperbolic system (again see [12]), giving the following.

Corollary 3.3. If $f: M \rightarrow M$ is weakly partially hyperbolic, $W^{c}$ and $W^{u}$ exist and are quasi-isometric, and the lifted foliation $W^{c}$ has polynomial growth of volume, then the universal cover has polynomial growth of volume.

Now consider strongly partially hyperbolic systems. Due to the three-way splitting $E^{s} \oplus E^{c} \oplus E^{u}$, we need to define a notion of product structure inside a foliation.

Definition. Suppose $\mathcal{F}$ and $\mathcal{G}$ are subfoliations of a foliation $\mathcal{W}$. Then $\mathcal{F}$ and $\mathcal{G}$ have (linear/polynomial) Global Product Structure inside $\mathcal{W}$ if they have this property when restricted to each leaf of $\mathcal{W}$. Any choice of polynomial must be independent of the leaf.

Most of the above results can be adapted to the case of product structure inside a foliation, with almost no change to the proofs.

Definition. Suppose a strongly partially hyperbolic system $f$ has foliations $W^{u}$, $W^{s}, W^{c}, W^{c u}$, and $W^{c s}$ tangent to $E^{u}, E^{s}, E^{c}, E^{c} \oplus E^{u}$, and $E^{c} \oplus E^{s}$. Then, $f$ has (linear/polynomial) Global Product Structure if the following pairs satisfy the property on the universal cover:
- $W^{c s}$ and $W^{u}$,
- $W^{s}$ and $W^{c u}$,
- $W^{s}$ and $W^{c}$ inside $W^{c s}$,
- $W^{c}$ and $W^{u}$ inside $W^{c u}$.

The following is proved, but not explicitly stated in [12].

Proposition 3.4. A strongly partially hyperbolic diffeomorphism with quasiisometric stable, center, and unstable foliations has linear Global Product Structure.

The next two results may be proved from their weakly partially hyperbolic counterparts.

Proposition 3.5. If $f: M \rightarrow M$ is strongly partially hyperbolic with linear Global Product Structure, and the lifted foliation $W^{c}$ has polynomial growth of volume, then the universal cover has polynomial growth of volume.

Corollary 3.6. If $f: M \rightarrow M$ is strongly partially hyperbolic, $W^{s}, W^{c}$ and $W^{u}$ exist and are quasi-isometric, and the lifted foliation $W^{c}$ has polynomial growth of volume, then the universal cover has polynomial growth of volume. 
Note that one-dimensional foliations always have polynomial growth of volume. One-dimensional center foliations are by far the most commonly studied case.

We now prove the last claim of the introduction.

Proof of Theorem 1.3. By classical Bieberbach theory, any diffeomorphism on a finite quotient of the 3-torus lifts to a diffeomorphism on the 3-torus [10]. For partial hyperbolicity on the 3-torus, it is already known that the invariant foliations exist and are quasi-isometric ([4, [11]).

For the other direction, apply Corollary [3.6 to see that the fundamental group has polynomial growth. In this case, $M$ is finitely covered by a circle bundle over a 2torus [16. For partially hyperbolic systems on such manifolds, the center foliations have been completely classified ([1], [13]). The center foliation is quasi-isometric if and only if the circle bundle is the 3 -torus.

\section{Appendix A. Proofs on Rectangles}

Proof of Lemma 2.1. As $E^{s}$ is uniformly continuous, there is $\epsilon_{0}>0$ such that $d_{c}(x, y)<\frac{4}{3} d(x, y)$ when $x$ and $y$ are points on the same local stable leaf and $d(x, y)<\epsilon_{0}$. Let $\sigma$ and $\mu$ be as in the definition of an Anosov diffeomorphism and let $\alpha>0$ be such that $\left\|T_{x} f^{-1}\right\|<\alpha$ for all $x$.

Given $R>0$, let $n$ be the smallest positive integer such that $\epsilon:=\mu^{-n} R$ satisfies the inequalities $3 \epsilon<\epsilon_{0}$ and $4 \epsilon \sigma^{n}<1$. Define $\delta:=\alpha^{-n} \epsilon$. Suppose $\phi$ is an $R$ rectangle for the ordering $\left(W^{u}, W^{s}\right)$, and that $\sup _{t} d_{s}(\phi(0,0), \phi(t, 0))<\delta$. Then, $f^{-n} \phi$ is an $\epsilon$-rectangle, $\sup _{t} d_{s}\left(f^{-n} \phi(0,0), f^{-n} \phi(t, 0)\right)<\epsilon$, and

$$
\begin{aligned}
d\left(f^{-n} \phi(0,1), f^{-n} \phi(1,1)\right)<3 \epsilon & \Rightarrow d_{s}\left(f^{-n} \phi(0,1), f^{-n} \phi(1,1)\right)<4 \epsilon \\
& \Rightarrow d_{s}(\phi(0,1), \phi(1,1))<1 .
\end{aligned}
$$

As $\delta=\delta(R)=\alpha^{-n} \mu^{-n} R$ and $n$ is proportional to $\log R$, there is a polynomial $p$ such that $1 / p(R)<\delta(R)$ for all $R>0$.

Proof of Lemma 3.1. For the ordered pair $\left(W^{c}, W^{u}\right)$ the proof is as in the Anosov case. For $\left(W^{u}, W^{c}\right)$, consider an $R$-rectangle $\phi$ with $d_{u}(\phi(0,1), \phi(1,1))>1$. We wish to bound $d_{u}(\phi(0,0), \phi(0,1))$ from below. By a compactness argument, there are constants $\gamma, \mu, \beta$ such that

$$
\left\|T f v^{c}\right\|<\gamma<\mu<\left\|T f v^{u}\right\|<\beta
$$

for all unit vectors $v^{c} \in E^{c}$ and $v^{u} \in E^{u}$. Then, $d_{u}\left(f^{n} \phi(0,1), f^{n} \phi(1,1)\right)>\mu^{n}$ for all $n>0$. By linear Global Product Structure, there is $n$ proportional to $\log R$ such that $d\left(f^{n} \phi(0,0), f^{n} \phi(1,1)\right)>2 \gamma^{n} R$. Then

$$
\begin{aligned}
d\left(f^{n} \phi(1,0), f^{n} \phi(1,1)\right)<\gamma^{n} R & \Rightarrow d\left(f^{n} \phi(0,0), f^{n} \phi(1,0)\right)>\gamma^{n} R \\
& \Rightarrow d(\phi(0,0), \phi(1,0))>\beta^{-n} \gamma^{n} R .
\end{aligned}
$$

As $n$ is proportional to $\log R$, this gives a polynomial bound.

\section{Appendix B. One Direction of Theorem 1.1}

Suggested steps in proving the "only if" direction of Theorem 1.1

Proposition B.1. If two Anosov diffeomorphisms are topologically conjugate and one has polynomial Global Product Structure, both do. 
Lemma B.2. Let $G$ be a nilpotent group with a subset $V$ such that $[V, V] \subset V$. Any element of the form $g=s_{1} u_{1} \cdots s_{n} u_{n}$ with $s_{i}, u_{i} \in V$ may be rewritten as

$$
g=s_{1} \cdots s_{n} a_{1} \cdots a_{\ell} u_{1} \cdots u_{n}
$$

where $a_{k} \in[V, V]$ for $k=1, \ldots, \ell$ and where $\ell$ depends polynomially on $n$.

Proposition B.3. If $f$ is a hyperbolic automorphism of a nilpotent Lie group $G$, then the stable and unstable foliations have polynomial Global Product Structure.

Proof. By Lemma B.2, reduce the problem from $f$ to $\left.f\right|_{[G, G]}$, which is also a hyperbolic automorphism of a nilpotent Lie group. Then apply induction.

\section{Appendix C. The proof of Theorem 2.5}

The proof in [5] is given in three steps. Step 1 constructs an infranilmanifold automorphism $g: P \rightarrow P$ covered by an automorphism $A$ of a nilpotent Lie group. This relies on an erroneous result of Auslander [1, Theorem 2]. In fact, the most we can say a priori is that $g$ is covered by an affine map $\alpha$ on a nilpotent Lie group (see [7, Theorem 2.2 and Definition 3.6] and also [14]).

To construct the automorphism $A$ from the affine map $\alpha$, we first show that $\alpha$ has a fixed point. Note that if a map $f:\left(M, x_{0}\right) \rightarrow\left(M, x_{0}\right)$ has a lift to the universal cover $\tilde{f}:\left(\tilde{M}, \tilde{x}_{0}\right) \rightarrow\left(\tilde{M}, \tilde{x}_{0}\right)$ such that $\tilde{x}_{0}$ is the unique fixed point, then the homomorphism $f_{*}$ induced on $\pi_{1}(M)$ satisfies $f_{*}(\gamma)=\gamma$ only when $\gamma$ is the identity. For an Anosov map with Global Product Structure, if $\tilde{f}$ had distinct fixed points $x, y \in \tilde{M}$, then $[x, y]$ would also be fixed. This would give two distinct fixed points on either the same stable or unstable leaf, a contradiction. Thus $f_{*}(\gamma) \neq \gamma$ for non-trivial $\gamma \in \pi_{1}(M)$. By [7, Lemmas 4.3 and 4.1], $\alpha$ does not have 1 as an eigenvalue and it has a unique fixed point. By adapting the proof of [7. Theorem 4.5], we may replace the infranilmanifold by one diffeomorphic to it, and replace the affine map $\alpha$ by a Lie group automorphism $A$. Then, Steps 2 and 3 of [5] follow as before.

\section{ACKNOWLEDGEMENTS}

The author would like to thank Charles Pugh, Rafael Potrie, and Amie Wilkinson for helpful comments.

\section{REFERENCES}

[1] Louis Auslander, Bieberbach's theorems on space groups and discrete uniform subgroups of Lie groups, Ann. of Math. (2) 71 (1960), 579-590. MR0121423 (22 \#12161)

[2] M. I. Brin, Nonwandering points of Anosov diffeomorphisms, Dynamical systems, Vol. I-Warsaw, Astérisque, No. 49, Soc. Math. France, Paris, 1977, pp. 11-18. MR0516499 $(58$ \#24378)

[3] M. Brin, On the fundamental group of a manifold admitting a U-diffeomorphism, Soviet Math. Dokl. 19 (1978), 497-500.

[4] Michael Brin, Dmitri Burago, and Sergey Ivanov, Dynamical coherence of partially hyperbolic diffeomorphisms of the 3-torus, J. Mod. Dyn. 3 (2009), no. 1, 1-11, DOI 10.3934/jmd.2009.3.1. MR2481329 (2010b:37084)

[5] M. Brin and A. Manning, Anosov diffeomorphisms with pinched spectrum, Dynamical systems and turbulence, Warwick 1980 (Coventry, 1979/1980), Lecture Notes in Math., vol. 898, Springer, Berlin, 1981, pp. 48-53. MR654882 (83j:58094)

[6] Keith Burns and Amie Wilkinson, Dynamical coherence and center bunching, Discrete Contin. Dyn. Syst. 22 (2008), no. 1-2, 89-100, DOI 10.3934/dcds.2008.22.89. MR2410949 (2010a:37055) 
[7] Karel Dekimpe, What an infra-nilmanifold endomorphism really should be. ., Topol. Methods Nonlinear Anal. 40 (2012), no. 1, 111-136. MR3026104

[8] John Franks, Anosov diffeomorphisms, Global Analysis (Proc. Sympos. Pure Math., Vol. XIV, Berkeley, Calif., 1968), Amer. Math. Soc., Providence, R.I., 1970, pp. 61-93. MR0271990 (42 \#6871)

[9] Mikhael Gromov, Groups of polynomial growth and expanding maps, Inst. Hautes Études Sci. Publ. Math. 53 (1981), 53-73. MR623534 (83b:53041)

[10] Ku Yong Ha, Jang Hyun Jo, Seung Won Kim, and Jong Bum Lee, Classification of free actions of finite groups on the 3-torus, Topology Appl. 121 (2002), no. 3, 469-507, DOI 10.1016/S0166-8641(01)00090-6. MR.1909004 (2003j:57056)

[11] Andrew Scott Hammerlindl, Leaf conjugacies on the torus, PhD Thesis, University of Toronto (Canada), 2009, ProQuest LLC, Ann Arbor, MI. MR2736718

[12] Andy Hammerlindl, Dynamics of quasi-isometric foliations, Nonlinearity 25 (2012), no. 6, 1585-1599, DOI 10.1088/0951-7715/25/6/1585. MR2924724

[13] Andy Hammerlindl, Partial hyperbolicity on 3-dimensional nilmanifolds, Discrete Contin. Dyn. Syst. 33 (2013), no. 8, 3641-3669. MR3021374

[14] Kyung Bai Lee and Frank Raymond, Rigidity of almost crystallographic groups, Combinatorial methods in topology and algebraic geometry (Rochester, N.Y., 1982), Contemp. Math., vol. 44, Amer. Math. Soc., Providence, RI, 1985, pp. 73-78, DOI 10.1090/conm/044/813102. MR813102(87d:57026)

[15] Anthony Manning, There are no new Anosov diffeomorphisms on tori, Amer. J. Math. 96 (1974), 422-429. MR0358865 (50 \#11324)

[16] Kamlesh Parwani, On 3-manifolds that support partially hyperbolic diffeomorphisms, Nonlinearity 23 (2010), no. 3, 589-606, DOI 10.1088/0951-7715/23/3/009. MR 2586372 (2011f:37056)

[17] Michael Shub, Expanding maps, Global Analysis (Proc. Sympos. Pure Math., Vol. XIV, Berkeley, Calif., 1968), Amer. Math. Soc., Providence, R.I., 1970, pp. 273-276. MR0266251 (42 \#1158)

School of Mathematics and Statistics, University of Sydney, Sydney, NSW 2006 , Australia 